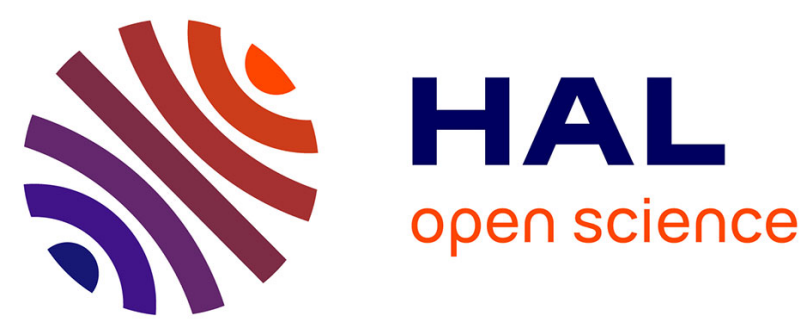

\title{
PERTINENCE DE L'ESSAI D'IMPACT DU CYLINDRE DE TAYLOR POUR LA PRÉVISION DU COMPORTEMENT BALISTIQUE DES ACIERS À BLINDAGE
}

M. Roux, M. Moureaud

\section{To cite this version:}

M. Roux, M. Moureaud. PERTINENCE DE L'ESSAI D'IMPACT DU CYLINDRE DE TAYLOR POUR LA PRÉVISION DU COMPORTEMENT BALISTIQUE DES ACIERS À BLINDAGE. Journal de Physique IV Proceedings, 1991, 01 (C3), pp.C3-271-C3-276. 10.1051/jp4:1991338 . jpa00250480

\author{
HAL Id: jpa-00250480 \\ https://hal.science/jpa-00250480
}

Submitted on 1 Jan 1991

HAL is a multi-disciplinary open access archive for the deposit and dissemination of scientific research documents, whether they are published or not. The documents may come from teaching and research institutions in France or abroad, or from public or private research centers.
L'archive ouverte pluridisciplinaire HAL, est destinée au dépôt et à la diffusion de documents scientifiques de niveau recherche, publiés ou non, émanant des établissements d'enseignement et de recherche français ou étrangers, des laboratoires publics ou privés. 


\title{
PERTINENCE DE L'ESSAI D' IMPACT DU CYLINDRE DE TAYLOR POUR LA PRÉVISION DU COMPORTEMENT BALISTIQUE DES ACIERS 'ं BLINDAGE
}

\author{
M.R. ROUX* et M.J. MOUREAUD \\ *Creusot-Loire Industrie, Division Creusot-Marrel, Centre de \\ Recherche des Matériaux du Creusot. BP. 56. F-71202 Le Creusot \\ cedex, France
}

Les modèles analytiques développés pour prédire le comportement balistique des aciers à blindage nécessitent la connaissance de la contrainte d'écoulement dynamique. Elle peut être mesurée à l'aide de l'essai d'impact du cylindre de Taylor car les vitesses de déformation atteintes sont proches de celles observées lors d'un tir balistique.

Nous avons validé cette approche sur des aciers à blindage à très haute dureté, Mars $270(550 \mathrm{HB})$ et Mars $300(600 \mathrm{HB})$.

AbstractIn order to predict the ballistic behavior of armor steels with analytical models, dynamic flow stress of the materials is needed. We can measure it by a Taylor dynamic impact test with deformation speed close to those observed in ballistic impact. We used this average dynamic flow stress to predict the ballistic behavior of two very high hard armor steels.

\section{1 - INTRODUCTION}

Comme il n'existe pas de relation directè entre les propriétés mécaniques d'un matériau et ses performances balistiques, la définition de nouvelles nuances passait, jusqu'à présent, par des essais balistiques nécessitant une quantité importante de matière.

Nous proposons ici une approche prévisionnelle du comportement balistique à l'aide d'essais d'impacts dynamiques ne nécessitant que peu de matière.

\section{2 - PRESENTATION DE LA METHODE}

La prévision du comportement balistique des aciers à blindage, en fonction des caractéristiques intrinsèques du couple cible-projectile (voir figure 1) repose méthodologiquement sur la compréhension de :

- la dynamique du blindage, par l'étude du comportement dynamique sous sollicitations à grande vitesse de déformation au moyen de machines à choc;

- la mécanique de la perforation, par l'analyse physique des mécanismes de pénétration et de perforation du blindage rencontrés au cours de limpact projectile/cible ;

- la modélisation de la perforation, par l'intermédiaire de modèles rhéologiques permettant de déterminer l'énergie absorbée lors du choc. 
Cette approche permet de montrer que la détermination de la résistance à la perforation d'une plaque en acier à blindage, impactée par un projectile perforant classique, ne dépend que de la contrainte d'écoulement dynamique de l'acier.

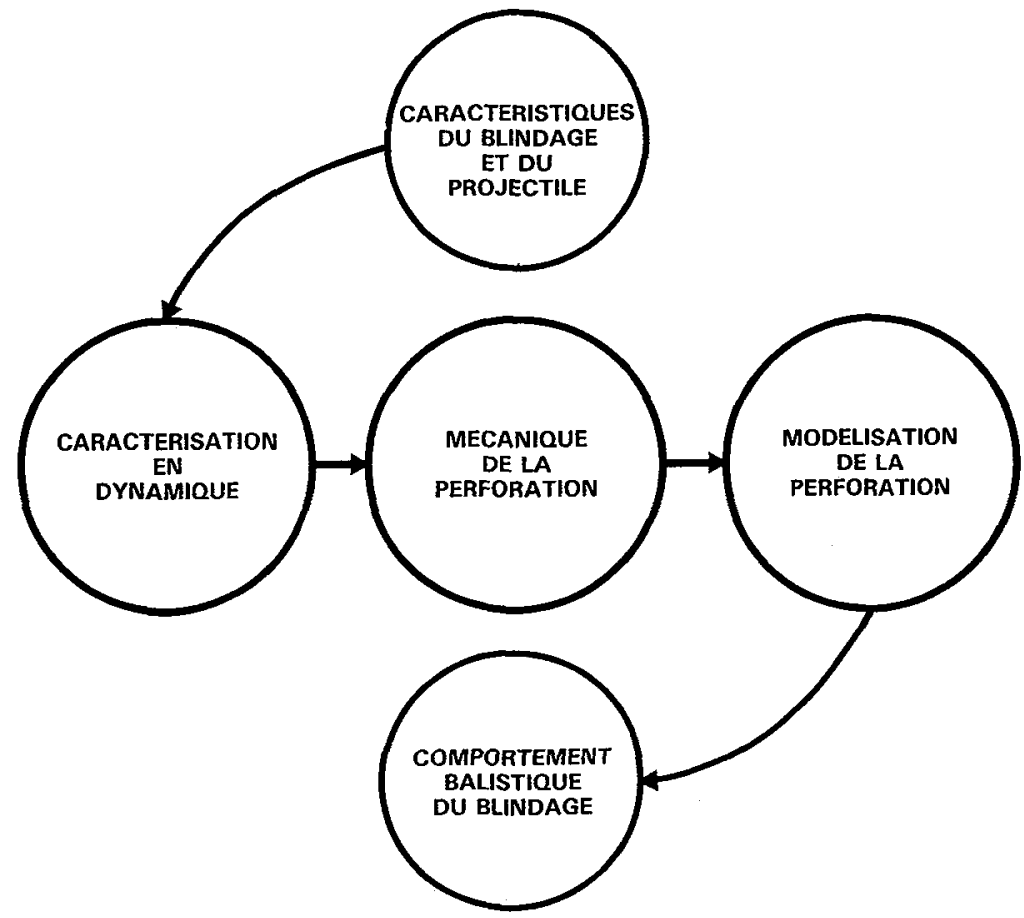

FIGURE 1:

METHODOLOGIE

\section{3 - CARACTERISATION EN DYNAMIOUE}

\section{1 - Movens expérimentaux}

L'essai dynamique le plus simple se rapprochant des vitesses de déformation atteintes lors d'un impact balistique est l'essai d'impact du cylindre de Taylor permettant d'obtenir une valeur moyenne de la contrainte d'écoulement.

Nous disposons d'un canon à gaz léger permettant de lancer des projectiles cylindriques de quelques grammes à des vitesses comprises entre 50 et $500 \mathrm{~m} / \mathrm{s}$ (voir figure 2).

Les projectiles sont guidés dans le tube par des sabots en plastique qui sont arrêtés par un extracteur avant impact sur une cible en carbure de tungstène supposếe indéformable.

La mesure de la vitesse du projectile se fait en comptant le temps de passage devant un faisceau laser juste avant impact. 

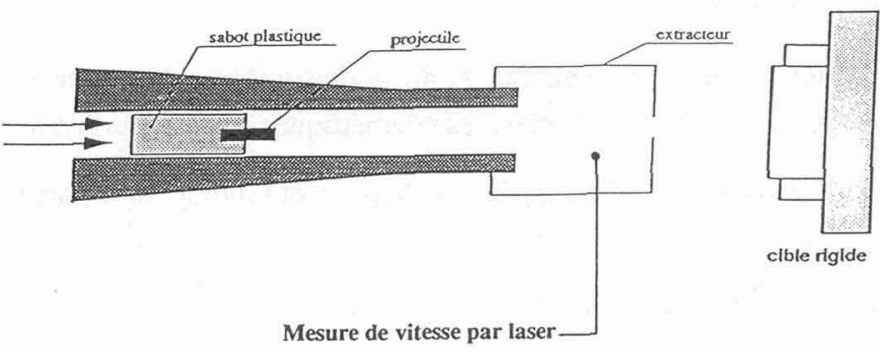

FIGURE 2:

PRINCIPE DE L'INSTALLATON D'ESSAI

DYNAMIQUE D'MMPACT DE TAYLOR

\section{2 - Dépouillement des essais}

Lors de l'impact, si le niveau de contrainte engendré dépasse la limite d'élasticité du matériau, le cylindre se déforme plastiquement en prenant l'aspect d'un "pied d'éléphant" (voir figure 3).
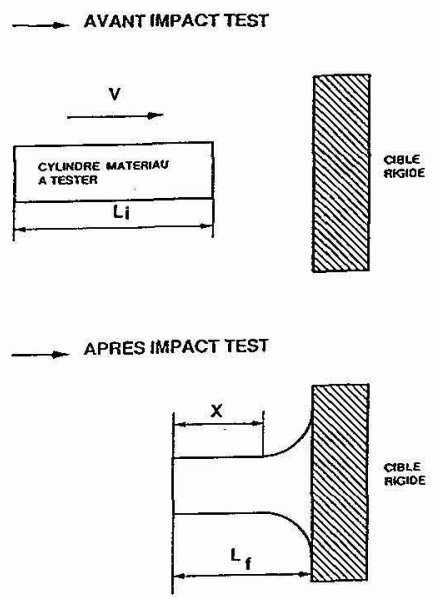

FIGURE 3:

EXPLOTTATION DE L'ESSAI PAR

MESURES GEOMETRIQUES 
Pour que l'essai soit exploitable, il est nécessaire d'assurer une très bonne perpendicularité entre la face impactée et l'axe du cylindre ; cela impose un alignement rigoureux de l'installation et une rectification soignée des éprouvettes.

La détermination de la contrainte d'écoulement $Y$, de la déformation moyenne $\varepsilon$ et de la vitesse moyenne de déformation $\dot{\varepsilon}$ se fait à partir des mesures géométriques de l'échantillon avant et après tir.

Nous avons choisi d'utiliser l'analyse développée par Wilkins et Guinan /1/ à partir de simulations numériques.

$$
\begin{aligned}
& Y=-\left(\rho V^{2} / 2\right) / \ln [(L f / L i-0,12) / 0,88] \\
& \varepsilon=-\ln (L f-X / L i-X) \\
& \dot{\varepsilon}=-(V / 2(L i-L f)) \cdot \ln (L f-X / L i-X)
\end{aligned}
$$

en désignant par :

$\rho$ la masse volumique du matériau à tester

$V$ la vitesse d'impact

$\mathrm{Li}$ et $\mathrm{Lf}$ les longueurs initiale et finale du cylindre

$X$ la longueur de la partie non déformée.

\section{3 - Résultats obtenus}

Nous avons caractérisé trois nuances d'aciers à blindage, un acier Très Haute Dureté Mars 240 (HB $~$ 500 ) et deux aciers de dureté supérieure Mars 270 (HB $\simeq 550$ ) et Mars 300 (HB $\simeq 600$ ).

Ces trois aciers présentent une structure martensitique faiblement revenue ( $\theta$ détensionnement $\sim 200^{\circ} \mathrm{C}$ ).

Les résultats sont présentés à la figure 4 ; on note que la contrainte d'écoulement ne dépend pas de la vitesse de déformation, nous utiliserons donc par la suite la valeur moyenne :

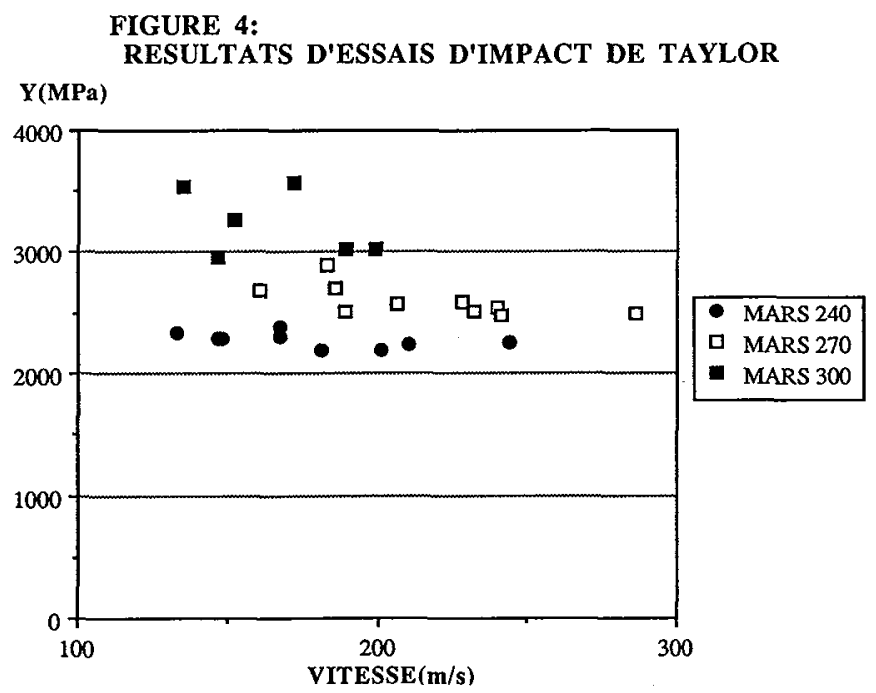


$\mathrm{Y} 240=2275 \mathrm{MPa}$

$\mathrm{Y} 270=2600 \mathrm{MPa}$

$\mathrm{Y} 300=3230 \mathrm{MPa}$

\section{4 - MODELISATION DE LA PERFORATION}

L'observation de cibles après tir montre un endommagement avec formation d'assiette arrière pour les trois matériaux (voir figure 5).

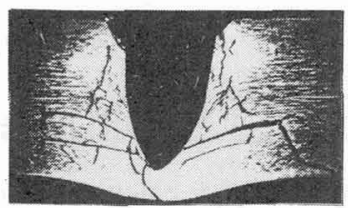

FIGURE 5:

EXEMPLE DE PERFORATION AVEC FORMATION

D'ASSIETTE ARRIERE

ACIER MARS 240 EPAISSEUR 20mm (HB $=505)$

PROJECTILE 12.7P $i=0^{\circ}$

Nous avons donc choisi l'analyse développée par Woodward /2/ pour exprimer le travail de perforation (voir figure 6).

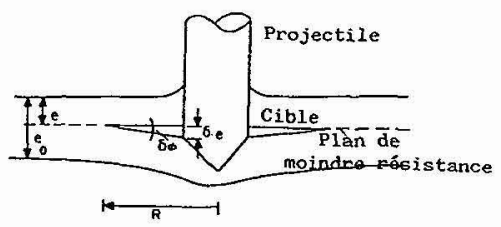

(1) DEBUt DE PENETRATION PAR ECOULEMENT PLASTIQUE RADIAL

(2) EXTENSIOÑ D'UNE FISSURE DANS UN PLAN DE HOINDRE RESISTANCE DE LA PLAQUE

(3) CHANGEMENT DU MODE DE PERFORATION

(4) FORMATION D'ASSIETTE ARRIERE

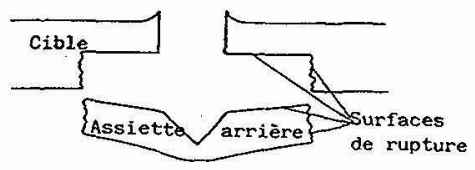

FIGURE 6: MODELISATION DE WOODWARD 
L'égalité entre l'énergie cinétique incidence et l'énergie nécessaire à la perforation permet d'écrire :

$$
\mathrm{VLP}^{2}=\pi \mathrm{d}^{2} \mathrm{Y}(\mathrm{e}-\mathrm{x}) / \mathrm{m}
$$

le paramètre $\mathrm{x}$ fonction du projectile et non du materiau à blindage sera déterminée par régression statistique sur les résultats relatifs au Mars 240.

Pour le projectile perforant calibre 12.7

$$
\begin{aligned}
& \mathrm{d}=10,9 \mathrm{~mm} \\
& \mathrm{~m}=26,9 \mathrm{~g} \\
& \mathrm{x} \text { vaut } 5,4 \mathrm{~mm} .
\end{aligned}
$$

\section{5 - PREVISION DU COMPORTEMENT BALISTIOUE}

La figure 7 montre le bon accord entre les VLP calculées à partir des essais dynamiques et de la modélisation choisie, et les résultats expérimentaux obtenus.

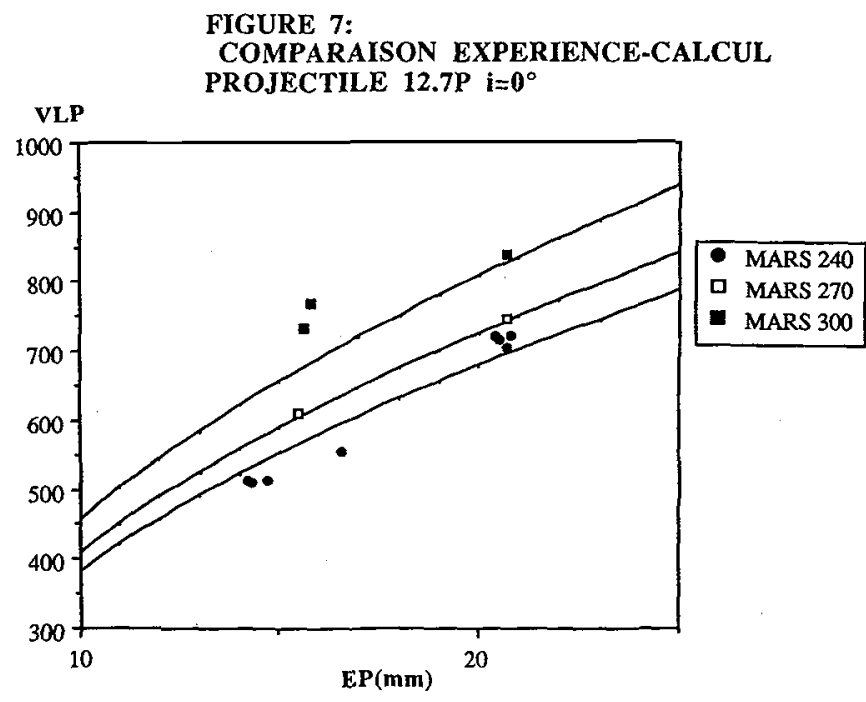

\section{6 - CONCLUSION}

L'exemple étudié montre qu'il est possible d'estimer les performances balistiques d'un nouvel acier à blindage à partir d'une caractérisation du matériau en dynamique. L'essai d'impact du cylindre de Taylor a été choisi car il se rapproche des vitesses de déformation atteintes lors d'un essai balistique.

Cet essai permet donc de développer de nouvelles nuances d'acier à blindage à partir de coulées expérimentales de quelques kilogrammes.

/1/ Impact of cylinders on a rigid boundary M.L. Wilkins, M.N. Guinan

J. Appl. Phys. Vol. $44 \mathrm{~N}^{\circ} 3$ - March 1973.

12/ The interrelation of failure modes observed in the penetration of metallic targets R.L. Woodward Int. J. Impact Engin. 1984. 